\title{
Translation Strategies of Figurative Language in the Novel The Fault in Our Stars
}

\author{
Deby Angelia \\ English Department, Faculty of Languages and Literature, Petra Christian University, Siwalankerto 121- \\ 131, Surabaya 60236, INDONESIA \\ Email:m11415036@john.petra.ac.id
}

\begin{abstract}
This research wanted to help the reader to understand about the classification of translation strategies in the novel The Fault in Our Stars. The writer used Larson's (1998), proposes three strategies to translate figurative language. The writer was interested in analyzing the figurative language because there are many kinds of implicit meaning in figurative language; she felt that it was interesting to be analyzed. Besides, the writer chose a novel because it explains the story more detail than others such as movie. She chose The Fault in Our Stars novel because the story is quite touched and there are a lot of figurative languages on its novel. The writer hope that the translated meaning of figurative language can be the same as the original text.
\end{abstract}

Keywords: Translation, Translation Strategy, Figurative Language, Source Language, Target Language.

\section{INTRODUCTION}

The Fault in Our Stars, is the sixth novel written by John Green. This novel tells the story of a sixteen-year-old girl named Hazel Grace who had cancer and spread to her lungs, who was forced by her parents to attend a support group. Later, she met and fell in love with Augustus Waters, a former basketball player and amputated. Unfortunately, the ending of this story is not a pleasant story.

There are so many figurative languages on the both novels such as "you're such a Debbie Downer" which refers to "someone who frequently bringing down the mood of everyone around them", etc. The purpose of the study is to find about the strategies used to translate the figurative language in the novel The Fault in Our Stars. Besides, she also wants to find the appropriateness in dominant strategy used by the translator to translate The Fault in Our Stars novel.

The main theory that I used is literal translation by Larson (2000). He states that the purpose of a translator in translating figurative language is to maintain the three elements of clarity, forth and beauty in the language. It can be said that the translation strategy is used by the translator to find out the problem solving in the process of translation.

According to Larson (2000), "Translation consists of transferring without distortion the meaning of the source language into the target language, meaning which must be kept constant, even when the form of the source language changes as it is turned into the form of the target language." By this statement, we learn that the impression by native speaker of the source language should be equivalent with the impression by native speaker of the target language.

According to Barbara (2014), "Figurative language is a broad range of concepts including metaphor, metonymy, simile, blending, and develops new tools for analyzing them." In translation, it is important to understand about what the figurative language is because many creations such as novel use figurative language in their story. If the translator cannot understand 
the meaning of figurative language in the source text and fails to analyze it correctly, there will be a misunderstanding because not all figurative language can be easily interpreted. Therefore, translator must be able to use appropriate words from the source text into the target text.

As a student who has learned about translation in the fifth semester, the writer chose to analyze the translation strategies of figurative language in the novel The Fault in Our Stars by John Green as well as the figurative language translation in the novel The Fault in Our Stars of Indonesian version by Ingrid Dwijani Nimpoeno (2012) for her research. The writer chose a novel as her thesis because she thought that novel is the most detail information worked compare to others; such as a movie, a magazine, etc. Also, she chose a novel entitled The Fault in Our Stars because she interested on its story. The story tells about a-sixteen-year-old girl named Hazel Grace who fights against her cancer.

By conducting a research on this topic, the writer hopes that this research would give knowledge to recognize and to determine the strategies in translating figurative language. Furthermore, the writer hopes that this research would be useful for those who want to conduct further studies in translation field.

\section{REVIEW OF RELATED LITERATURE}

\section{Review of Related Theories}

Larson (2000) states that "the purpose of a translator in translating figurative language is to maintain the three elements of clarity, forth and beauty in the language". It can be said that the translation strategy is used by the translator to find out the problem solving in the process of translation. Larson (2000) proposes three strategies to translate figurative language.

A. The sense of the word may be translated non-figuratively

The translator changed the literal meaning from the source language into the literal meaning in the target language so that the readers of the target language are able to feel the sense of figurative language from the source language. For example:

Source text: Even if you are there, even if you are not there, and you fell law, they ate you (Larson, 2000, p.170)

B. Retaining the word in the original, but to add the sense of the word

The translator changed the word of the source text into the common word that often used by the readers of target text. However, the sense for both source language and target language is similar. For example:

Source text: The baby's nose was running(Larson, 2000, p.177)

C. Substituting a figurative expression of the target language for the figurative expression of the source language

The translator did not change or replace to any similar word because the figurative language in the source language has the same meaning as the figurative language in the target language. For example:

Source text: The wealthy live here(Larson, 2000. P.176) 


\section{Review of Related Studies}

\section{The Choice of Larson's Translation Strategies and the Resulted Quality of Indonesian Translation of Hyperboles in The Shrunken Head Novel (Oktaviani, 2018)}

The writer chose Oktaviani's research as her related studies because she had the same topic as the writer which dealt with translation strategies. Oktaviani wanted to reveal the common strategy used of hyperbole in The Shrunken Head novel. Oktaviani used Larson's (1998), Newmark's (1988) theories in order to classify the translation strategies of hyperbole that she had found in the novel she used. In conclusion, Oktaviani found that the translation strategy of retaining the word in the original, but to add the sense of the word became the most strategy that used by the translator in translating hyperboles in The Shrunken Head novel. She also found that the analysis of the level of accuracy in translating hyperbole was $88 \%$ accurate which means the messages of hyperbole of the source language is successfully conveyed to the target language.

The differences between the writer's and Oktaviani's research were the writer not only found out about the hyperbole in translation strategies, but also the other types of figurative languages such as personification, metaphor, simile, etc. Besides, the writer did not do research whether or not the figurative language used in the source language was accurate as the figurative language in the target language. The writer used Larson's theory in order to show what types of translation strategies used in The Fault in Our Stars novel and what the common strategy often used by the translator in The Fault in Our Stars novel. In this research, the writer classifying eight figurative languages and finds what translation strategies are used in each figurative language. Also, she counts manually the translation strategy that is often used by the translator to translate The Fault in Our Stars novel.

\section{Translation Errors in English-Indonesian Humor Text Produced by Student of Basic Translation Class (Gunawan, 2013)}

The writer chose Gunawan's research as her related studies because she used the same order as the writer. Gunawan wanted to reveal about what kinds of errors are found in the translation produced by basic translation student and what kind of error is mostly produced by the student. Gunawan used Newmark's, and Munday's theories in order to find the most frequent kind of error which is made by student. In conclusion, Gunawan found that the most occurs error is Change One Phrase of the Story. The differences between the writer and Gunawan's research were the writer used Larson's theory, whereas Gunawan used Newmark's and Munday's theory for translation errors. Also, the writer analyzed the data use three majors' classification by Larson's and Gunawan used two major types to analyze her data. The similarities between the writer and Gunawan's research were both used English to Indonesian translation and had the same purpose to deliver the same meaning from the source language into the meaning of the target language.

From Gunawan's research, the writer learned something about how to make an interesting and effective table which she put on her thesis. Gunawan's table inspired writer to create the similar one. The following table is taken from Gunawan's thesis in chapter three. 
Gunawan's table

\begin{tabular}{|l|l|l|l|l|l|l|}
\hline No. & English Text & $\begin{array}{l}\text { Translated Text } \\
\text { (Indonesian) }\end{array}$ & $\begin{array}{l}\text { Suggested } \\
\text { correction }\end{array}$ & E & PV & Notes \\
\hline 1.1 & & & & & & \\
\hline 1.2 & & & & & & \\
\hline 2.1 & & & & & &
\end{tabular}

\section{METHOD}

In this research, the writer used a quantitative method. According to Aliaga and Gunderson (2002), this research is quantitative that means “... an inquiry into a social problem, explain phenomena by gathering numerical data that are analyzed using mathematically based method". The writer analyzed English and Indonesian sentences which contain figurative languages, then she describes the strategies of each data. This research design was applied in order to make the research easy and successful. Research method can be interpreted as a device or procedure used in order to collect, process, analyze and present data or solve the problems. Research method is basically a way you can use to achieve a goal. In this research, the writer used quantitative method. She collected the data of figurative languages and translation strategies, then she counted the number of how many figurative languages found on each translation strategy.

The instrument of the study is the writer collected and analyzed the data, then counted it at the end to find out the dominant strategy in the novel The Fault in Our Stars. First, she read the origin novel of The Fault in Our Stars by John Green. Second, she re- read and at the same time collected the figurative language based on each chapter. The writer gave a mark on each figurative language in every chapter, so it will be easier when she collects all figurative language becomes one table. Third, the writer also read the translated novel to get figurative language. She collected the data from the translated novel using the similar way when she collected the figurative language in the origin novel. Fourth, the writer compared between both figurative languages in the source text and in the target text and put them on the table. The source of data is from two novels entitled The Fault in Our Stars (John Green, 2012 and Dwijani Nimpoeno, 2012). There are twenty-five chapters in the novel, but the writer just analyzed chapter 1-15. From fifteen chapters, there are approximately 60-80 figurative language sentences. The writer chose chapter 1-15 because according to the author, John Green, one of the lessons from this novel is at the beginning of the novel which explains about how the author writes authentically from a 16-year-old girl's perspective. The writer will classify whether the translation of figurative language is appropriate with the origin novel or not, she also will describe about the strategy used in the translated novel. Total of the pages from the origin novel is 313 pages and 422 pages for the translated novel.

In data analysis, the writer divided the data as source text (figurative language in English novel) and target text (figurative language in Indonesian or translated novel). She also put FL (figurative language) on the table in order to classify the FL from both novels. Also, she put TS (translation strategy) as well as notes to tell and describe the strategy used of the target text. Below is the table of writer's data analysis. The writer gave code $(1.1 .1,1.1 .2,2.1 .1,3.1 .1$, etc.) to differentiate the chapter, the figurative language, and how many examples on a table. "1.1.1" means "chapter 1, figurative language 1, example 1", "1.1.2" means "chapter 1, figurative language 1, example 2", 
"2.1.1" means "chapter 2, figurative language 1, example 1", "3.1.1" means "chapter 3 , figurative language 1, example 1", etc. After analyzing and put the data on the table, the writer manually counted the number of translation strategies from all chapters in order to get the idea of the dominant strategy often used in The Fault in Our Stars novel.

\section{FINDINGS AND ANALYSIS}

In analyzing the data, the writer classified the analysis into three parts. The first part is figurative languages which include in the sense of the word may be translated non-figuratively, the second part is figurative languages which include retaining the word in the original but add the sense of the word, and the third part is figurative languages which include substituting a figurative expression of the target language for the figurative expression of the source language. The writer also classified each table by its chapter and figurative language. There are seven figurative languages and each figurative language has their own number. For personification, the writer put it as figurative language 1. For metaphor, the writer put it as figurative language 2. For simile, the writer put it as figurative language 3. For hyperbole, the writer put it as figurative language 4. For paradox, the writer put it as figurative language 5. For irony, the writer put it as figurative language 6. For synecdoche, the writer put it as figurative language 7. On the table, the writer will put a number for the chapter. Then, following by the number of figurative language. The last is the number for the example.

\section{A. The sense of the word may be translated non-figuratively}

In this part, the writer puts figurative language before the original and translated texts. Then, she puts translation strategy as well as notes for the explanation. In this part, the translation strategy of each figurative language uses the sense of the word may be translated non-figuratively. Here the writer will divide discussion into four categories: Translation Strategy in Metaphor, Translation Strategy in Simile, Translation Strategy in Synecdoche, and Translation Strategy in Hyperbole.

Table 4.1.1 Translation Strategy in Metaphor

\begin{tabular}{|c|l|l|l|l|l|}
\hline No. & Page & English Text & Indonesian Text & \multicolumn{1}{|c|}{$\begin{array}{l}\text { Appropriate / } \\
\text { Inappropriate }\end{array}$} \\
\hline 1.2 .1 & $4 ; 10$ & $\begin{array}{l}\text { Doctor Jim } \\
\text { agreed that I } \\
\text { was veritably } \\
\text { swimming in a } \\
\text { paralyzing and } \\
\text { totally clinical } \\
\text { depression }\end{array}$ & $\begin{array}{l}\text { Dokter Jim } \\
\text { mengiyakan } \\
\text { bahwa aku jelas } \\
\text { berkubang } \\
\text { dalam depresi } \\
\text { yang } \\
\text { melumpuhkan } \\
\text { dan benar- } \\
\text { benar klinis }\end{array}$ & $\begin{array}{l}\text { "Swimming" has different } \\
\text { meaning to "berkubang". In } \\
\text { Indonesia, "berkubang" has } \\
\text { related to "lumpur". While, } \\
\text { this content refers to a girl } \\
\text { whom depressed because of } \\
\text { her illness. }\end{array}$ & Inappropriate \\
\hline 1.2 .2 & $7 ; 14$ & $\begin{array}{l}\text { Television is a } \\
\text { passivity }\end{array}$ & $\begin{array}{l}\text { Televisi adalah } \\
\text { pasivitas }\end{array}$ & $\begin{array}{l}\text { "Passivity" in Indonesian } \\
\text { means "kepasifan". While, } \\
\text { "pasivitas" has no definition } \\
\text { in Indonesia Dictionary. In } \\
\text { this content, Hazel's mom } \\
\text { tried to give explanation that } \\
\text { "you cannot interact or make } \\
\text { friends with a television" } \\
\text { because television is a passive } \\
\text { stuff. }\end{array}$ & Inappropriate \\
\hline 8.2 .1 & $114 ;$ \\
155 & $\begin{array}{l}\text { She was a } \\
\text { hugger }\end{array}$ & $\begin{array}{l}\text { The translator changed the } \\
\text { word "hugger" into "senang } \\
\text { memeluk" which has different }\end{array}$ & Inappropriate \\
\hline
\end{tabular}




\begin{tabular}{|l|l|l|l|l|l|}
\hline & & $\begin{array}{l}\text { meaning from the original } \\
\text { novel. }\end{array}$ & \\
\hline
\end{tabular}

Table 4.1.2 Translation Strategy in Simile

\begin{tabular}{|c|l|l|l|l|l|}
\hline No. & Page & English Text & Indonesian Text & \multicolumn{1}{|c|}{ Notes } & $\begin{array}{l}\text { Appropriate / } \\
\text { Inappropriate }\end{array}$ \\
\hline 1.3 .1 & $11 ;$ & $\begin{array}{l}\text { She said that } \\
\text { she felt strong, } \\
\text { which felt like } \\
\text { bragging to me } \\
\text { as the oxygen- } \\
\text { drizzling nubs } \\
\text { tickled my } \\
\text { nostrils }\end{array}$ & $\begin{array}{l}\text { Dia merasa } \\
\text { kuat, dan } \\
\text { bagiku ini } \\
\text { terasa seperti } \\
\text { menyombongka } \\
\text { n diri, } \text { ketika } \\
\text { ujung-ujung } \\
\text { selang penyalur } \\
\text { oksigen } \\
\text { menggelitiki } \\
\text { lubang } \\
\text { hidungku }\end{array}$ & $\begin{array}{l}\text { What Hazel meant was, the } \\
\text { girl who said that she was } \\
\text { strong, actually she was not as } \\
\text { strong as its reality. The } \\
\text { translator changed the } \\
\text { meaning from English text to } \\
\text { Indonesian text. }\end{array}$ & Appropriate \\
\hline 5.3 .1 & $\begin{array}{l}76 ; \\
106\end{array}$ & $\begin{array}{l}\text { And then, like } \\
\text { the bitch I am }\end{array}$ & $\begin{array}{l}\text { Lalu, } \text { karena } \\
\text { aku memang } \\
\text { menyebalkan }\end{array}$ & $\begin{array}{l}\text { The translator changed the } \\
\text { word "bitch" into } \\
\text { "menyebalkan" but the } \\
\text { Indonesian readers did not get } \\
\text { the same meaning as the } \\
\text { native English readers. }\end{array}$ & Inappropriate \\
\hline 11.3 .1 & $\begin{array}{l}156 ; \\
211\end{array}$ & $\begin{array}{l}\text { I could see } \\
\text { dozens of } \\
\text { houseboats } \\
\text { moored along } \\
\text { the water, it } \\
\text { looked like an } \\
\text { old painting }\end{array}$ & $\begin{array}{l}\text { Aku melihat } \\
\text { lusinan rumah- } \\
\text { kapal berlabuh } \\
\text { di sepanjang } \\
\text { perairan }\end{array}$ & $\begin{array}{l}\text { The translator omitted the } \\
\text { word "looked like an old } \\
\text { painting" so the Indonesian } \\
\text { readers did not get the same } \\
\text { meaning as the native English } \\
\text { readers. }\end{array}$ & Inappropriate \\
\hline
\end{tabular}

Table 4.1.3 Translation Strategy in Synecdoche

\begin{tabular}{|l|l|l|l|l|l|}
\hline No. & Page & English Text & Indonesian Text & \multicolumn{1}{|c|}{ Notes } & $\begin{array}{l}\text { Appropriate / } \\
\text { Inappropriate }\end{array}$ \\
\hline 3.7 .1 & $42 ;$ & $\begin{array}{l}\text { Kaitlyn saw me } \\
\text { the moment I } \\
\text { raised my hand, } \\
\text { flashed her } \\
\text { very white and } \\
\text { newly } \\
\text { straightened } \\
\text { teeth at me }\end{array}$ & $\begin{array}{l}\text { Begitu aku } \\
\text { mengangkat } \\
\text { tangan, dia } \\
\text { langsun } \\
\text { melihatku, } \\
\text { memamerkan } \\
\text { giginya yang } \\
\text { putih bersih } \\
\text { dan baru saja } \\
\text { diluruskan }\end{array}$ & $\begin{array}{l}\text { The translator changed the } \\
\text { word "flashed" into } \\
\text { "memamerkan". Both of two } \\
\text { words are different and the } \\
\text { meaning of both texts was also } \\
\text { different. }\end{array}$ & Appropriate \\
\hline
\end{tabular}

Table 4.1.4 Translation Strategy in Hyperbole

\begin{tabular}{|c|l|l|l|l|l|}
\hline No. & Page & English Text & Indonesian Text & \multicolumn{1}{|c|}{ Notes } & $\begin{array}{l}\text { Appropriate / } \\
\text { Inappropriate }\end{array}$ \\
\hline 10.4 .1 & $141 ;$ & $\begin{array}{l}\text { After about ten } \\
\text { seconds, my } \\
\text { lungs felt like } \\
\text { they were } \\
\text { folding in upon } \\
\text { themselves like } \\
\text { flowers at dusk }\end{array}$ & $\begin{array}{l}\text { Setelah } \text { kira- } \\
\text { kira sepuluh } \\
\text { detik, paru- } \\
\text { paruku terasa } \\
\text { seakan terlipat- } \\
\text { lipat seperti } \\
\text { bunga di } \text { waktu } \\
\text { senja }\end{array}$ & $\begin{array}{l}\text { The translator changed the } \\
\text { word "folding in upon } \\
\text { themselves" into "terlipat- } \\
\text { lipat" which has different } \\
\text { meaning. In case, the } \\
\text { Indonesian readers did not get } \\
\text { the same meaning as the } \\
\text { native English readers. }\end{array}$ & Appropriate \\
\hline
\end{tabular}




\section{B. Retaining the word in the original, but to add the sense of the word}

In this part, the writer puts figurative language before the original and translated texts. Then, she puts translation strategy as well as notes for the explanation. In this part, the translation strategy of each figurative language uses retaining the word in the original, but to add the sense of the word. Here the writer will divide discussion into seven categories: Translation Strategy in Hyperbole, Translation Strategy in Paradox, Translation Strategy in Irony, Translation Strategy in Synecdoche, Translation Strategy in Metaphor, Translation Strategy in Personification, and Translation Strategy in Simile.

Table 4.2.1 Translation Strategy in Hyperbole

\begin{tabular}{|c|c|c|c|c|c|}
\hline No. & Page & English Text & Indonesian Text & Notes & $\begin{array}{l}\text { Appropriate / } \\
\text { Inappropriate }\end{array}$ \\
\hline 1.4 .1 & $4 ; 11$ & $\begin{array}{l}\text { We sat down in } \\
\text { the Circle of } \\
\text { Trust and } \\
\text { listened to } \\
\text { Patrick recount } \\
\text { for the } \\
\text { thousandth } \\
\text { time his } \\
\text { depressingly } \\
\text { miserable life } \\
\text { story }\end{array}$ & $\begin{array}{l}\text { Kami duduk } \\
\text { membentuk } \\
\text { Lingkaran } \\
\text { Kepercayaan } \\
\text { dan untuk } \\
\text { kesekian kalinya } \\
\text { mendengarkan } \\
\text { Patrick } \\
\text { menceritakan } \\
\text { kembali kisah } \\
\text { hidupnya yang } \\
\text { menyedihkan } \\
\text { dan membuat } \\
\text { depresi }\end{array}$ & $\begin{array}{l}\text { In the novel, Patrick was not } \\
\text { re-telling his miserable story } \\
\text { for "thousandth time". The } \\
\text { author just tried to exaggerate } \\
\text { the meaning. Then, the } \\
\text { translator translated "recount } \\
\text { for the thousandth time" into } \\
\text { "menceritakan kembali kisah } \\
\text { hidupnya" which is different } \\
\text { for literal translation. The } \\
\text { translator chose its sentence in } \\
\text { order to enhance the sense of } \\
\text { Indonesian readers. }\end{array}$ & Appropriate \\
\hline 3.4 .1 & $\begin{array}{l}44 \\
63\end{array}$ & $\begin{array}{l}\text { Then she } \\
\text { grabbed a pair } \\
\text { of strappy } \\
\text { hooker shoes } \\
\text { and said, "Is it } \\
\text { even possible to } \\
\text { walk in these? I } \\
\text { mean, I would } \\
\text { just die-" }\end{array}$ & $\begin{array}{l}\text { Lalu, dia meraih } \\
\text { sepasang sepatu } \\
\text { bertali dan } \\
\text { berhak } \\
\text { supertinggi dan } \\
\text { berkata, } \\
\text { "Bisakah } \\
\text { berjalan } \\
\text { dengan sepatu } \\
\text { ini? Maksudku, } \\
\text { lebih baik aku } \\
\text { mati saja-" }\end{array}$ & $\begin{array}{l}\text { "To walk in there?" and } \\
\text { "berjalan dengan sepatu ini?" } \\
\text { are different. However, the } \\
\text { translator changed it so it was } \\
\text { not complicated. }\end{array}$ & Appropriate \\
\hline 11.4 .1 & $\begin{array}{l}166 \\
224\end{array}$ & $\begin{array}{l}\text { I was going to } \\
\text { have to go } \\
\text { through hell } \\
\text { for six months } \\
\text { or a year }\end{array}$ & $\begin{array}{l}\text { Aku harus } \\
\text { menjalani } \\
\text { neraka selama } \\
\text { enam bulan } \\
\text { atau setahun }\end{array}$ & $\begin{array}{l}\text { The translator changed the } \\
\text { word "go through" into } \\
\text { "menjalankan". However, the } \\
\text { Indonesian readers still get the } \\
\text { same meaning as the native } \\
\text { English readers. }\end{array}$ & Inappropriate \\
\hline
\end{tabular}

Table 4.2.2 Translation Strategy in Paradox

\begin{tabular}{|c|c|l|l|l|c|}
\hline No. & Page & English Text & Indonesian Text & \multicolumn{1}{|c|}{$\begin{array}{l}\text { Nppropriate / } \\
\text { Inappropriate }\end{array}$} \\
\hline 1.5 .1 & $5 ; 11$ & $\begin{array}{l}\text { Slowly working } \\
\text { his way toward } \\
\text { a master's } \\
\text { degree that will } \\
\text { not improve his } \\
\text { career prospects }\end{array}$ & $\begin{array}{l}\text { Perlahan-lahan } \\
\text { berupaya } \\
\text { meraih gelar } \\
\text { master yang } \\
\text { tidak akan } \\
\text { meningkatkan }\end{array}$ & $\begin{array}{l}\text { "Working" in Indonesian } \\
\text { means "bekerja"; in progress } \\
\text { to achieve master's degree. } \\
\text { Whereas, "berupaya" is more } \\
\text { likely to "trying". However, } \\
\text { the meaning of the two texts is } \\
\text { same for both readers. }\end{array}$ & Appropriate \\
\hline
\end{tabular}




\begin{tabular}{|l|l|l|l|l|}
\hline & & $\begin{array}{l}\text { prospek } \\
\text { kariernya }\end{array}$ & & \\
\hline
\end{tabular}

Table 4.2.3 Translation Strategy in Irony

\begin{tabular}{|c|c|c|c|c|c|}
\hline No. & Page & English Text & Indonesian Text & Notes & $\begin{array}{l}\text { Appropriate / } \\
\text { Inappropriate }\end{array}$ \\
\hline 1.6 .1 & $8 ; 15$ & $\begin{array}{l}\text { There is only } \\
\text { one thing in this } \\
\text { world shittier } \\
\text { than biting it } \\
\text { from cancer } \\
\text { when you're } \\
\text { sixteen, and } \\
\text { that's having a } \\
\text { kid who bites it } \\
\text { from cancer }\end{array}$ & $\begin{array}{l}\text { Hanya ada satu } \\
\text { hal di dunia ini } \\
\text { yang lebih } \\
\text { menyebalkan } \\
\text { daripada mati } \\
\text { gara-gara } \\
\text { kanker di usia } \\
\text { enam belas, } \\
\text { yaitu punya } \\
\text { anak yang mati } \\
\text { gara-gara } \\
\text { kanker }\end{array}$ & $\begin{array}{l}\text { "Shittier, biting, bites" are not } \\
\text { literally translated to } \\
\text { Indonesian text by the } \\
\text { translator. However, both of } \\
\text { English text and Indonesian } \\
\text { text have the same meaning } \\
\text { for their own readers. }\end{array}$ & Appropriate \\
\hline 1.6 .2 & $\begin{array}{l}15 \\
26\end{array}$ & $\begin{array}{l}\text { I feel so } \\
\text { fortunate that } \\
\text { an intellectual } \\
\text { giant like } \\
\text { yourself would } \\
\text { deign to } \\
\text { operate on me }\end{array}$ & $\begin{array}{l}\text { Aku merasa } \\
\text { sangat } \\
\text { beruntung } \\
\text { karena orang } \\
\text { yang } \\
\text { mahacerdas } \\
\text { seperti dirimu } \\
\text { bersedia } \\
\text { mengoperasiku }\end{array}$ & $\begin{array}{l}\text { In fact, Issac was not really } \\
\text { feel fortunate; instead torture. } \\
\text { The translator changed the } \\
\text { words "intellectual giant" into } \\
\text { "mahacerdas" so that the } \\
\text { Indonesian readers can get the } \\
\text { same meaning as native } \\
\text { English readers. }\end{array}$ & Appropriate \\
\hline 9.6 .1 & $\begin{array}{l}130 \\
176\end{array}$ & $\begin{array}{l}\text { I've gotten } \\
\text { really hot since } \\
\text { you went blind }\end{array}$ & $\begin{array}{l}\text { Aku menjadi } \\
\text { sangat seksi } \\
\text { semenjak kau } \\
\text { buta }\end{array}$ & $\begin{array}{l}\text { The translator omitted the } \\
\text { word "went" in Indonesian } \\
\text { text. However, both of readers } \\
\text { can et the same meaning. }\end{array}$ & Appropriate \\
\hline
\end{tabular}

Table 4.2.4 Translation Strategy in Synecdoche

\begin{tabular}{|c|c|l|l|l|c|}
\hline No. & Page & English Text & Indonesian Text & \multicolumn{1}{|c|}{ Notes } & $\begin{array}{l}\text { Appropriate / } \\
\text { Inappropriate }\end{array}$ \\
\hline 1.7 .1 & $9 ; 1$ & $\begin{array}{l}\text { I cut a glance to } \\
\text { him, and his } \\
\text { eyes were still } \\
\text { on me }\end{array}$ & $\begin{array}{l}\text { Aku melirik } \\
\text { cowok itu, dan } \\
\text { matanya masih } \\
\text { terpaku padaku }\end{array}$ & $\begin{array}{l}\text { "Were still on me" and } \\
\text { "terpaku padaku" have the } \\
\text { same meaning even though } \\
\text { not translated literally. }\end{array}$ & Appropriate \\
\hline
\end{tabular}

Table 4.2.5 Translation Strategy in Metaphor

\begin{tabular}{|c|l|l|l|l|l|}
\hline No. & Page & English Text & Indonesian Text & \multicolumn{1}{|c|}{ Notes } & $\begin{array}{l}\text { Appropriate / } \\
\text { Inappropriate }\end{array}$ \\
\hline 1.2 .1 & $11 ;$ & $\begin{array}{l}\text { I'm on a roller } \\
\text { coaster that } \\
\text { only goes up, } \\
\text { my friend }\end{array}$ & $\begin{array}{l}\text { Serasa berada } \\
\text { di atas roller } \\
\text { coaster yang } \\
\text { hanya melesat } \\
\text { ke atas, sobatku }\end{array}$ & $\begin{array}{l}\text { "I'm on a roller coaster" has } \\
\text { different meaning to "Serasa } \\
\text { berada di atas roller coaster". } \\
\text { However, the translator tried } \\
\text { to make Indonesian readers } \\
\text { get the same meaning as } \\
\text { native English readers. }\end{array}$ & Appropriate \\
\hline 2.2 .1 & $27 ;$ & $\begin{array}{l}\text { You're such a } \\
\text { Debbie Downer }\end{array}$ & $\begin{array}{l}\text { Kau benar- } \\
\text { benar merusak } \\
\text { suasana }\end{array}$ & $\begin{array}{l}\text { The English text and the } \\
\text { Indonesian text are really not } \\
\text { literally translated. However, } \\
\text { the translator is quite good to } \\
\text { understand the meaning of } \\
\text { English text. She translated } \\
\text { into the same meaning but }\end{array}$ & Appropriate \\
\hline
\end{tabular}




\begin{tabular}{|c|c|c|c|c|c|}
\hline & & & & $\begin{array}{l}\text { different sentence so that the } \\
\text { Indonesian readers can get the } \\
\text { same meaning as native } \\
\text { English readers. }\end{array}$ & \\
\hline 2.2 .2 & $\begin{array}{l}36 ; \\
53\end{array}$ & $\begin{array}{l}\text { He really was } \\
\text { beautiful }\end{array}$ & $\begin{array}{l}\text { Dia sungguh } \\
\text { rupawan }\end{array}$ & $\begin{array}{l}\text { The translator changed the } \\
\text { word "beautiful" into } \\
\text { "rupawan" because she does } \\
\text { not want to make the } \\
\text { Indonesian text becomes } \\
\text { complicated because of } \\
\text { Hazel's statement. }\end{array}$ & Inappropriate \\
\hline 3.2 .1 & $\begin{array}{l}42 ; \\
61\end{array}$ & $\begin{array}{l}\text { I've been dating } \\
\text { Derek } \\
\text { Wellington for a } \\
\text { bit, but I don't } \\
\text { think it will last. } \\
\text { He's such a boy }\end{array}$ & $\begin{array}{l}\text { Aku mengencani } \\
\text { Derek } \\
\text { Wellington } \\
\text { untuk sementara } \\
\text { ini, tapi kurasa } \\
\text { tidak akan lama. } \\
\text { Dia begitu ke- } \\
\text { kanak-kanak- } \\
\text { an }\end{array}$ & $\begin{array}{l}\text { The translator changed the } \\
\text { word "such a boy" into "ke- } \\
\text { kanak-kanak-an" because it is } \\
\text { easy to be understood by the } \\
\text { Indonesian readers. }\end{array}$ & Appropriate \\
\hline 8.2 .1 & $\begin{array}{l}116 ; \\
158\end{array}$ & $\begin{array}{l}\text { They might be } \\
\text { glad to have me } \\
\text { around, but I } \\
\text { was the alpha } \\
\text { and the omega } \\
\text { of my parents' } \\
\text { suffering }\end{array}$ & $\begin{array}{l}\text { Mereka } \\
\text { mungkin senang } \\
\text { memilikiku, tapi } \\
\text { akulah awal } \\
\text { dan akhir dari } \\
\text { penderitaan } \\
\text { orang tuaku }\end{array}$ & $\begin{array}{l}\text { The translator is quite good to } \\
\text { understand the metaphor used } \\
\text { here. She changed the } \\
\text { sentence "alpha and omega" } \\
\text { into "awal dan akhir" and } \\
\text { they have the same meaning } \\
\text { for both readers. }\end{array}$ & Appropriate \\
\hline
\end{tabular}

Table 4.2.6 Translation Strategy in Personification

\begin{tabular}{|c|c|c|c|c|c|}
\hline No. & Page & English Text & Indonesian Text & Notes & $\begin{array}{l}\text { Appropriate / } \\
\text { Inappropriate }\end{array}$ \\
\hline 3.1 .1 & $\begin{array}{l}42 ; \\
60\end{array}$ & $\begin{array}{l}\text { I do wish you } \\
\text { were at school } \\
\text { these days. } \\
\text { Some of the } \\
\text { boys have } \\
\text { become } \\
\text { downright } \\
\text { edible }\end{array}$ & $\begin{array}{l}\text { Aku benar- } \\
\text { benar berharap } \\
\text { kau ada di } \\
\text { sekolah } \\
\text { belakangan ini. } \\
\text { Beberapa } \\
\text { cowok sudah } \\
\text { berubah } \\
\text { menjadi sangat } \\
\text { menggiurkan }\end{array}$ & $\begin{array}{l}\text { The translator changed the } \\
\text { word "edible" into } \\
\text { "menggiurkan" so that the } \\
\text { Indonesian readers can get the } \\
\text { same meaning as the native } \\
\text { English readers. }\end{array}$ & Inappropriate \\
\hline 5.1 .1 & $\begin{array}{l}65 \\
91\end{array}$ & $\begin{array}{l}\text { Of course made } \\
\text { me worry that } \\
\text { my lungs were } \\
\text { again } \\
\text { swimming in a } \\
\text { rising pool of } \\
\text { fluid }\end{array}$ & $\begin{array}{l}\text { Tentu saja ini } \\
\text { membuatku } \\
\text { khawatir paru- } \\
\text { paruku sekali } \\
\text { lagi berenang } \\
\text { dalam } \\
\text { genangan } \\
\text { cairan yang } \\
\text { semakin banyak }\end{array}$ & $\begin{array}{l}\text { "Rising pool" literally has } \\
\text { different meaning to } \\
\text { "genangan". However, } \\
\text { "genangan" is still can be } \\
\text { accepted or related to "kolam } \\
\text { renang". So, the translator } \\
\text { changed it in order to make } \\
\text { the Indonesian readers get the } \\
\text { same meaning as the native } \\
\text { English readers. }\end{array}$ & Inappropriate \\
\hline 10.1 .1 & $\begin{array}{l}142 \\
192\end{array}$ & $\begin{array}{l}\text { The pain was } \\
\text { always there, } \\
\text { demanding to } \\
\text { be felt }\end{array}$ & $\begin{array}{l}\text { Rasa nyeri itu } \\
\text { selalu ada di } \\
\text { sana, menuntut } \\
\text { untuk } \\
\text { dirasakan }\end{array}$ & $\begin{array}{l}\text { The translator changed the } \\
\text { literal meaning of the word } \\
\text { "pain" into "nyeri". However, } \\
\text { the Indonesian readers still get } \\
\text { the same meaning as the } \\
\text { native English readers. }\end{array}$ & Appropriate \\
\hline
\end{tabular}


Table 4.2.7 Translation Strategy in Simile

\begin{tabular}{|c|c|c|c|c|c|}
\hline No. & Page & English Text & Indonesian Text & Notes & $\begin{array}{l}\text { Appropriate / } \\
\text { Inappropriate }\end{array}$ \\
\hline 4.3 .1 & $\begin{array}{l}60 \\
85\end{array}$ & $\begin{array}{l}\text { Issac nodded, } \\
\text { the tears not } \\
\text { like tears so } \\
\text { much as a quiet } \\
\text { metronome }\end{array}$ & $\begin{array}{l}\text { Issac } \\
\text { mengangguk, } \\
\text { air matanya } \\
\text { lebih } \\
\text { menyerupai } \\
\text { metronom yang } \\
\text { berdetak pelan }\end{array}$ & $\begin{array}{l}\text { The translator changed the } \\
\text { sentence in English text to } \\
\text { Indonesian text with easier } \\
\text { way. She omitted "not like } \\
\text { tears" which means "tidak } \\
\text { seperti air mata" in Indonesia. } \\
\text { She just tried to make the } \\
\text { sentence become not } \\
\text { complicated. }\end{array}$ & Inappropriate \\
\hline 7.3 .1 & $\begin{array}{l}110 \\
150\end{array}$ & $\begin{array}{l}\text { Thanks for not } \\
\text { trying to see me } \\
\text { when I looked } \\
\text { like hell }\end{array}$ & $\begin{array}{l}\text { Terima kasih } \\
\text { karena tidak } \\
\text { berupaya } \\
\text { menengokku } \\
\text { ketika } \\
\text { penampilanku } \\
\text { berantakan }\end{array}$ & $\begin{array}{l}\text { The translator changed the } \\
\text { word "hell" into } \\
\text { "berantakan". However, both } \\
\text { of readers get the same } \\
\text { meaning of reading TFIOS } \\
\text { novel either original or } \\
\text { translated. }\end{array}$ & Appropriate \\
\hline
\end{tabular}

\section{Substituting a figurative expression of the target language for the figurative expression of the source language}

In this part, the writer puts figurative language before the original and translated texts. Then, she puts translation strategy as well as notes for the explanation. In this part, the translation strategy of each figurative language uses substituting a figurative expression of the target language for the figurative expression of the source language. Here the writer will divide discussion into seven categories: Translation Strategy in Metaphor, Translation Strategy in Simile, Translation Strategy in Hyperbole, Translation Strategy in Synecdoche, Translation Strategy in Irony, Translation Strategy in Paradox, and Translation Strategy in Personification.

Table 4.3.1 Translation Strategy in Metaphor

\begin{tabular}{|c|l|l|l|l|c|}
\hline No. & Page & English Text & Indonesian Text & \multicolumn{1}{|c|}{ Notes } & $\begin{array}{l}\text { Appropriate / } \\
\text { Inappropriate }\end{array}$ \\
\hline 1.2 .1 & $\begin{array}{l}14 ; \\
23-\end{array}$ & $\begin{array}{l}\text { Lord Jesus } \\
\text { Christ, we are } \\
\text { gathered here } \\
\text { in Your heart, } \\
\text { literally in Your } \\
\text { heart }\end{array}$ & $\begin{array}{l}\text { Tuhan Yesus } \\
\text { Kristus, } \text { kami } \\
\text { berkumpul di } \\
\text { sini di dalam } \\
\text { jantung-Mu, } \\
\text { secara harafiah } \\
\text { di dalam } \\
\text { jantung-Mu }\end{array}$ & $\begin{array}{l}\text { They are not literally gathered } \\
\text { in Jesus's heart, but in the } \\
\text { church basement. The } \\
\text { translator literally translated it } \\
\text { because its metaphor is easy to } \\
\text { be understood for most } \\
\text { Indonesian readers. }\end{array}$ & Appropriate \\
\hline 6.2 .1 & $\begin{array}{l}99 ; \\
136\end{array}$ & $\begin{array}{l}\text { I'm a grenade } \\
\text { and at some } \\
\text { point I'm going } \\
\text { to blow up and } \\
\text { I would like to } \\
\text { minimize the } \\
\text { casualties }\end{array}$ & $\begin{array}{l}\text { Aku granat dan } \\
\text { suatu } \text { ketika } \\
\text { aku akan } \\
\text { meledak, } \\
\text { sehingga aku } \\
\text { ingin } \\
\text { meminimalkan } \\
\text { jumlah korban }\end{array}$ & $\begin{array}{l}\text { The translator literally } \\
\text { translated the sentence. } \\
\text { However, we know that it was } \\
\text { just a metaphor because of the } \\
\text { mix words between "I" and } \\
\text { "grenade" }\end{array}$ & Appropriate \\
\hline 8.2 .1 & $\begin{array}{l}121 ; \\
165\end{array}$ & $\begin{array}{l}\text { The sky is } \\
\text { depressing me }\end{array}$ & $\begin{array}{l}\text { Langitnya } \\
\text { membuatku } \\
\text { depresi }\end{array}$ & $\begin{array}{l}\text { The translator did not change } \\
\text { any words; we know that } \\
\text { "sky" cannot "depress" us. }\end{array}$ & Appropriate \\
\hline
\end{tabular}




\begin{tabular}{|l|l|l|l|l|l|}
\hline 10.2 .1 & $\begin{array}{l}141 ; \\
191\end{array}$ & $\begin{array}{l}\text { I was a } \\
\text { nonmetallicize } \\
\text { d creature }\end{array}$ & $\begin{array}{l}\text { Aku adalah } \\
\text { makhluk tak } \\
\text { berlogam }\end{array}$ & $\begin{array}{l}\text { The translator did not change } \\
\text { the sentence, we know it was } \\
\text { just a metaphor because "I" is } \\
\text { a human and there is no metal } \\
\text { human in life. }\end{array}$ & Appropriate \\
\hline 12.2 .1 & $195 ;$ & $\begin{array}{l}\text { His brain is } \\
\text { Swiss cheese. } \\
\text { He doesn't even } \\
\text { remember } \\
\text { writing the } \\
\text { book. }\end{array}$ & $\begin{array}{l}\text { Otaknya seperti } \\
\text { keju Swiss. Dia } \\
\text { bahkan tidak } \\
\text { ingat menulis } \\
\text { buku itu. }\end{array}$ & $\begin{array}{l}\text { The translator literally } \\
\text { translated the sentence, we } \\
\text { know the meaning of “Swiss } \\
\text { cheese" because of the next } \\
\text { sentence "doesn't even } \\
\text { remember". The Indonesian } \\
\text { readers get the same meaning } \\
\text { as native English readers. }\end{array}$ & Appropriate \\
\hline
\end{tabular}

Table 4.3.2 Translation Strategy in Simile

\begin{tabular}{|c|c|c|c|c|c|}
\hline No. & Page & English Text & Indonesian Text & Notes & $\begin{array}{l}\text { Appropriate / } \\
\text { Inappropriate }\end{array}$ \\
\hline 1.3 .1 & $6 ; 13$ & $\begin{array}{l}\text { Like his whole } \\
\text { head was } \\
\text { basically just } \\
\text { this fake eye } \\
\text { and this real } \\
\text { eye staring at } \\
\text { you }\end{array}$ & $\begin{array}{l}\text { Seakan seluruh } \\
\text { kepalanya bisa } \\
\text { dibilang hanya } \\
\text { terdiri dari } \\
\text { mata palsu dan } \\
\text { mata asli yang } \\
\text { menatapmu ini }\end{array}$ & $\begin{array}{l}\text { In fact, Issac's head was not } \\
\text { contain just fake eye and real } \\
\text { eye. The translator literally } \\
\text { translated the sentence from } \\
\text { English text to Indonesian } \\
\text { text. Besides, Indonesian } \\
\text { readers also can grasp the } \\
\text { same meaning as the native } \\
\text { English readers. }\end{array}$ & Appropriate \\
\hline 3.3 .1 & $\begin{array}{l}43 ; \\
62\end{array}$ & $\begin{array}{l}\text { Kaitlyn hated } \\
\text { her feet because } \\
\text { she felt her } \\
\text { second toes } \\
\text { were too long, } \\
\text { as if the second } \\
\text { toe was a } \\
\text { window into } \\
\text { the soul or } \\
\text { something }\end{array}$ & $\begin{array}{l}\text { Kaitlyn } \\
\text { membenci } \\
\text { kakinya karena } \\
\text { dia merasa } \\
\text { telunjuk kakinya } \\
\text { kepanjangan, } \\
\text { seakan telunjuk } \\
\text { kaki adalah } \\
\text { jendela untuk } \\
\text { melihat jiwa } \\
\text { atau } \\
\text { semacamnya }\end{array}$ & $\begin{array}{l}\text { The translator literally } \\
\text { translated and the sentence } \\
\text { was still can be accepted for } \\
\text { Indonesian readers. }\end{array}$ & Appropriate \\
\hline 6.3 .1 & $\begin{array}{l}99 ; \\
136\end{array}$ & $\begin{array}{l}\text { My dad titled } \\
\text { his head a little } \\
\text { to the side, like } \\
\text { a scolded } \\
\text { puppy }\end{array}$ & $\begin{array}{l}\text { Dad sedikit } \\
\text { memiringkan } \\
\text { kepala ke } \\
\text { samping, seperti } \\
\text { anak anjing } \\
\text { yang dimarahi }\end{array}$ & $\begin{array}{l}\text { The translator did not change } \\
\text { any words, but we know that it } \\
\text { was just a simile by the word } \\
\text { "like" }\end{array}$ & Appropriate \\
\hline 12.3 .1 & $\begin{array}{l}180 ; \\
243\end{array}$ & $\begin{array}{l}\text { His face and } \\
\text { belly were so } \\
\text { round, like a } \\
\text { dough ball }\end{array}$ & $\begin{array}{l}\text { Wajah dan } \\
\text { perutnya begitu } \\
\text { bulat, } \\
\text { menyerupai } \\
\text { bola adonan }\end{array}$ & $\begin{array}{l}\text { The translator literally } \\
\text { changed the sentence, we } \\
\text { know that it was just a simile } \\
\text { from the words comparison } \\
\text { "face and belly" and "a dough } \\
\text { ball" as well as the word } \\
\text { "like" between them. }\end{array}$ & Appropriate \\
\hline 12.3 .2 & $\begin{array}{l}196 ; \\
265\end{array}$ & $\begin{array}{l}\text { Lidewij drove a } \\
\text { clunky gray Fiat } \\
\text { with an engine } \\
\text { that sounded } \\
\text { like an excited }\end{array}$ & $\begin{array}{l}\text { Lidewij } \\
\text { menyetir Fiat } \\
\text { kelabu berisik } \\
\text { dengan mesin } \\
\text { yang } \\
\text { kedengarannya }\end{array}$ & $\begin{array}{l}\text { The translator literally } \\
\text { translated the sentence, we } \\
\text { know that it was just a smile } \\
\text { because the comparison words } \\
\text { of "engine" and "an excited }\end{array}$ & Appropriate \\
\hline
\end{tabular}




\begin{tabular}{|l|l|l|l|l|}
\hline four-year-old & $\begin{array}{l}\text { seperti anak } \\
\text { perempuan } \\
\text { empat tahun } \\
\text { yang } \\
\text { kegirangan }\end{array}$ & $\begin{array}{l}\text { four-year-old girl" as well as } \\
\text { the word "like" between them. }\end{array}$ & \\
\hline
\end{tabular}

Table 4.3.3 Translation Strategy in Hyperbole

\begin{tabular}{|c|c|c|c|c|c|}
\hline No. & Page & English Text & Indonesian Text & Notes & $\begin{array}{l}\text { Appropriate / } \\
\text { Inappropriate }\end{array}$ \\
\hline 2.4 .1 & $\begin{array}{l}25 ; \\
38\end{array}$ & $\begin{array}{l}\text { My father was } \\
\text { trying so hard } \\
\text { not to sob that } \\
\text { when he did, it } \\
\text { was an } \\
\text { earthquake }\end{array}$ & $\begin{array}{l}\text { Dad berupaya } \\
\text { begitu keras } \\
\text { untuk tidak } \\
\text { menangis } \\
\text { sehingga, ketika } \\
\text { dia menangis, } \\
\text { rasanya seperti } \\
\text { gempa bumi }\end{array}$ & $\begin{array}{l}\text { The translator literally } \\
\text { translated the sentence from } \\
\text { English text to Indonesian } \\
\text { text. For most Indonesian } \\
\text { readers, they will know that } \\
\text { the sentence is just hyperbole } \\
\text { by the word "seakan". }\end{array}$ & Appropriate \\
\hline 3.4 .1 & $\begin{array}{l}43 ; \\
61\end{array}$ & $\begin{array}{l}\text { Phalanxifor! So } \\
\text { you could just } \\
\text { live forever, } \\
\text { right? }\end{array}$ & $\begin{array}{l}\text { Phalanxifor! } \\
\text { Jadi, kau bisa } \\
\text { hidup untuk } \\
\text { selamanya, } \\
\text { bukan? }\end{array}$ & $\begin{array}{l}\text { The translator literally } \\
\text { translated the sentence, but we } \\
\text { all know that live is not } \\
\text { forever, it was just a } \\
\text { hyperbole. }\end{array}$ & Appropriate \\
\hline 4.4 .1 & $\begin{array}{l}48 ; \\
69\end{array}$ & $\begin{array}{l}\text { I started } \\
\text { reading An } \\
\text { Imperial } \\
\text { Affliction for } \\
\text { the millionth } \\
\text { time }\end{array}$ & $\begin{array}{l}\text { Aku mulai } \\
\text { membaca } \\
\text { Kemalangan } \\
\text { Luar Biasa } \\
\text { untuk kesejuta } \\
\text { kalinya }\end{array}$ & $\begin{array}{l}\text { The translator literally } \\
\text { translated the sentence, most } \\
\text { of Indonesian readers know } \\
\text { that millionth time was just a } \\
\text { hyperbole. }\end{array}$ & Appropriate \\
\hline 11.4 .1 & $\begin{array}{l}165 ; \\
222\end{array}$ & $\begin{array}{l}\text { I want this } \\
\text { dragon carrot } \\
\text { risotto to } \\
\text { become a } \\
\text { person so I can } \\
\text { take it to Las } \\
\text { Vegas and } \\
\text { marry it }\end{array}$ & $\begin{array}{l}\text { Aku ingin } \\
\text { risotto wortel- } \\
\text { naga ini } \\
\text { berubah } \\
\text { menjadi orang, } \\
\text { sehingga aku } \\
\text { bisa } \\
\text { membawanya } \\
\text { ke Las Vegas } \\
\text { dan } \\
\text { menikahinya }\end{array}$ & $\begin{array}{l}\text { The translator literally } \\
\text { translated the sentence and we } \\
\text { know that it was just a } \\
\text { hyperbole by the words } \\
\text { "marry it" or "marry the carrot } \\
\text { risotto". Carrot risotto is meal, } \\
\text { will never be a person. }\end{array}$ & Appropriate \\
\hline 12.4 .1 & $\begin{array}{l}196 ; \\
264\end{array}$ & $\begin{array}{l}\text { I'm not going } \\
\text { anywhere with } \\
\text { that monster }\end{array}$ & $\begin{array}{l}\text { Aku tidak akan } \\
\text { pergi kemana- } \\
\text { mana bersama } \\
\text { monster itu }\end{array}$ & $\begin{array}{l}\text { The translator literally } \\
\text { translated the sentence, we } \\
\text { know that it was just a } \\
\text { hyperbole by the word } \\
\text { "monster" which means in } \\
\text { literal word is "Peter Van } \\
\text { Houten" }\end{array}$ & Appropriate \\
\hline
\end{tabular}

Table 4.3.4 Translation Strategy in Synec

\begin{tabular}{|c|l|l|l|l|c|}
\hline No. & Page & English Text & Indonesian Text & \multicolumn{1}{|c|}{ Notes } & $\begin{array}{l}\text { Appropriate / } \\
\text { Inappropriate }\end{array}$ \\
\hline 1.7 .1 & $10 ;$ & $\begin{array}{l}\text { When he looked } \\
\text { back at me, I } \\
\text { flicked my } \\
\text { eyebrows up to } \\
\text { say, I win. }\end{array}$ & $\begin{array}{l}\text { Ketika dia } \\
\text { kembali } \\
\text { memandangku, } \\
\text { kunaikkan alis } \\
\text { untuk } \\
\text { mengatakan, } \\
\text { aku menang }\end{array}$ & $\begin{array}{l}\text { The author used parts of body } \\
\text { to express an implicit } \\
\text { meaning. The translator } \\
\text { translated it literally and the } \\
\text { Indonesian readers get the } \\
\text { same meaning as the native } \\
\text { English readers. }\end{array}$ & Appropriate \\
\hline
\end{tabular}


Table 4.3.5 Translation Strategy in Irony

\begin{tabular}{|c|l|l|l|l|l|}
\hline No. & Page & English Text & Indonesian Text & \multicolumn{1}{|c|}{$\begin{array}{l}\text { Appropriate / } \\
\text { Inappropriate }\end{array}$} \\
\hline 1.6 .1 & $15 ;$ & $\begin{array}{l}\text { Thank you for } \\
\text { explaining that } \\
\text { my eye cancer } \\
\text { isn't going to } \\
\text { make me deaf }\end{array}$ & $\begin{array}{l}\text { Terima } \text { kasih } \\
\text { atas } \\
\text { penjelasanmu } \\
\text { bahwa } \text { kanker } \\
\text { mata tidak akan } \\
\text { membuatku tuli }\end{array}$ & $\begin{array}{l}\text { Issac, a boy whom said that, } \\
\text { did not really mean to say } \\
\text { "thank you" to the nurse. He } \\
\text { wants to show his resentment } \\
\text { on his way. The translator } \\
\text { literally translated the } \\
\text { sentence because it has same } \\
\text { meaning as for the Indonesian } \\
\text { readers. }\end{array}$ & Appropriate \\
\hline 4.6 .1 & $\begin{array}{l}54 ; \\
76\end{array}$ & $\begin{array}{l}\text { So welcome to } \\
\text { the sweet } \\
\text { torture of } \\
\text { reading } \text { An } \\
\text { Imperial- }\end{array}$ & $\begin{array}{l}\text { Jadi, selamat } \\
\text { menikmati } \\
\text { siksaan manis } \\
\text { ketika membaca } \\
\text { Kemalangan } \\
\text { Luar Biasa- }\end{array}$ & $\begin{array}{l}\text { The translator literally } \\
\text { translated the sentence. } \\
\text { However, most of Indonesian } \\
\text { readers know that "sweet" and } \\
\text { "torture" are nonsense and } \\
\text { they were just an irony } \\
\text { because of the word } \\
\text { "welcome" }\end{array}$ & Appropriate \\
\hline 12.6 .1 & $\begin{array}{l}182 ; \\
246\end{array}$ & $\begin{array}{l}\text { It is at any rate a } \\
\text { pleasure to } \\
\text { meet such } \\
\text { ontologically } \\
\text { improbable } \\
\text { creatures }\end{array}$ & $\begin{array}{l}\text { Bagaimanapun, } \\
\text { senang } \\
\text { berjumpa } \\
\text { dengan } \\
\text { makhluk yang } \\
\text { secara ontologis } \\
\text { mustahil }\end{array}$ & $\begin{array}{l}\text { The translator literally } \\
\text { translated the sentence, we } \\
\text { know that it was an irony } \\
\text { because of the mix words of } \\
\text { "pleasure" and "ontologically } \\
\text { improbable creatures"; two } \\
\text { contradictive words. }\end{array}$ & Appropriate \\
\hline
\end{tabular}

Table 4.3.6 Translation Strategy in Paradox

\begin{tabular}{|c|c|l|l|l|c|}
\hline No. & Page & English Text & Indonesian Text & $\begin{array}{l}\text { Appropriate / } \\
\text { Inappropriate }\end{array}$ \\
\hline 3.5 .1 & $\begin{array}{l}45 ; \\
64\end{array}$ & $\begin{array}{l}\text { I think my } \\
\text { school friends } \\
\text { wanted to help } \\
\text { me through my } \\
\text { cancer, but they } \\
\text { eventually } \\
\text { found out that } \\
\text { they couldn't. }\end{array}$ & $\begin{array}{l}\text { Kurasa teman- } \\
\text { teman } \\
\text { sekolahku ingin } \\
\text { membantuku } \\
\text { mengatasi } \\
\text { kanker, tapi } \\
\text { pada akhirnya } \\
\text { mereka tahu } \\
\text { bahwa } \text { mereka } \\
\text { tidak bisa. }\end{array}$ & $\begin{array}{l}\text { The translator literally } \\
\text { translated the sentence and } \\
\text { most of Indonesian readers are } \\
\text { able to understand the purpose } \\
\text { of its saying. }\end{array}$ & Appropriate \\
\hline 6.5 .1 & $\begin{array}{l}93 ; \\
127\end{array}$ & $\begin{array}{l}\text { It all felt } \\
\text { Romantic, but } \\
\text { not romantic }\end{array}$ & $\begin{array}{l}\text { Semuanya } \\
\text { terasa } \\
\text { Romantis, tapi } \\
\text { tidak romantis }\end{array}$ & $\begin{array}{l}\text { The translator did not change } \\
\text { any words and it still can be } \\
\text { understood by Indonesian } \\
\text { readers. }\end{array}$ & Appropriate \\
\hline
\end{tabular}

Table 4.3.7 Translation Strategy in Personification

\begin{tabular}{|c|l|l|l|l|c|}
\hline No. & Page & English Text & Indonesian Text & \multicolumn{1}{|c|}{$\begin{array}{l}\text { Notes } \\
\text { Inappropriate / }\end{array}$} \\
\hline 5.1 .1 & $\begin{array}{l}64- \\
65 ; \\
91\end{array}$ & $\begin{array}{l}\text { We were seated } \\
\text { around our little } \\
\text { circular table in } \\
\text { the kitchen } \\
\text { when my phone } \\
\text { started singing }\end{array}$ & $\begin{array}{l}\text { Kami sedang } \\
\text { duduk di meja } \\
\text { melingkar kecil } \\
\text { kami di dapur } \\
\text { ketika ponselku } \\
\text { mulai menyanyi }\end{array}$ & $\begin{array}{l}\text { The translator literally } \\
\text { translated it, but we know that } \\
\text { "phone" is just a stuff; its } \\
\text { cannot be singing do } \\
\text { something which human does. }\end{array}$ & Inappropriate \\
\hline
\end{tabular}




\begin{tabular}{|l|l|l|l|l|l|}
\hline 11.1 .1 & $\begin{array}{l}167 ; \\
225\end{array}$ & $\begin{array}{l}\text { The sun was a } \\
\text { toddler } \\
\text { insistently } \\
\text { refusing to go } \\
\text { to bed: It was } \\
\text { past eight thirty } \\
\text { and still light }\end{array}$ & $\begin{array}{l}\text { Matahari } \\
\text { seperti bayi } \\
\text { yang bersikeras } \\
\text { menolak untuk } \\
\text { tidur: Saat itu } \\
\text { sudah lewat } \\
\text { pukul delapan } \\
\text { tiga puluh, tapi } \\
\text { hari masih } \\
\text { terang }\end{array}$ & $\begin{array}{l}\text { The translator literally } \\
\text { translated the sentence, we } \\
\text { know that "the sun" is not a } \\
\text { person so it should cannot be } \\
\text { "refusing" for something. }\end{array}$ & Appropriate \\
\hline 12.1 .1 & $195 ;$ & $\begin{array}{l}\text { I heard the } \\
\text { plonk plonk of } \\
\text { high heels } \\
\text { running }\end{array}$ & $\begin{array}{l}\text { Aku mendenagr } \\
\text { prok prok } \\
\text { sepatu tumit } \\
\text { tinggi berlari- } \\
\text { lari }\end{array}$ & $\begin{array}{l}\text { The translator literally } \\
\text { changed the sentence, we } \\
\text { know that it was just a } \\
\text { personification because "high } \\
\text { heels" have no power to } \\
\text { "running" like human does. }\end{array}$ & Appropriate \\
\hline 12.1 .2 & $\begin{array}{l}198- \\
199 ;\end{array}$ & $\begin{array}{l}\text { My lungs } \\
\text { telling my } \\
\text { brain } \text { oh, God, } \\
\text { we're dying } \\
\text { here }\end{array}$ & $\begin{array}{l}\text { Paru-paruku } \\
\text { mengatakan } \\
\text { pada otakku } \\
\text { astaga, } \text { kita } \\
\text { sekarat di } \text { sini }\end{array}$ & $\begin{array}{l}\text { The translator literally } \\
\text { translated the sentence, we } \\
\text { know that it was just a } \\
\text { personification because } \\
\text { "lungs" have no power to } \\
\text { "telling" like human does. }\end{array}$ & Appropriate \\
\hline 12.1 .3 & $\begin{array}{l}200 ; \\
270\end{array}$ & $\begin{array}{l}\text { The muscles in } \\
\text { my arms and } \\
\text { legs screaming } \\
\text { for oxygen }\end{array}$ & $\begin{array}{l}\text { Otot-otot } \\
\text { lengan dan } \\
\text { kakiku } \\
\text { berteriak } \\
\text { meminta } \\
\text { oksigen }\end{array}$ & $\begin{array}{l}\text { The translator literally } \\
\text { translated the sentence, we } \\
\text { know that it was just a } \\
\text { personification because “the } \\
\text { muscles" has no power to } \\
\text { "screaming" like human does. }\end{array}$ & Appropriate \\
\hline
\end{tabular}

\section{CONCLUSION}

In brief, the findings of her thesis: there are three strategies of translation, they are the sense of the word may be translated non-figuratively, retaining the word in the original but to add the sense of the word, substituting a figurative expression of the target language for the figurative expression of the source language. Also, the most appropriate translation is substituting a figurative expression of the target language for the figurative expression of the source language. From this thesis, the writer learned something about the differences between translating explicit text and implicit text (figurative language). Besides, the difficulty during the process of her thesis was she lacks of knowledge about the meaning of implicit words and sentences. In conclusion, the writer finally found an answer of the statement of problems for her thesis using experts' theories and the methodology. She found out the findings using Larson's three translation strategies. Last, the writer hopes this research about translation strategy in figurative language can help others who interested in this field to write similar topic with her.

\section{REFERENCES}

Larson, M. (2000). Meaning-based Translation. USA: $5^{\text {th }}$ edition, University Press of America. Barbara, D. (2014). Figurative Language. New York: University of British Columbia. Newmark, P. (1981). Approaches to translation. Oxford and New York: Perggamon Institute of English.

Green, J. (2012). The Fault in Our Stars. United States.

Nimpoeno, D (2012). The Fault in Our Stars. Indonesia.

Oktaviani, N. (2018). The Choice of Larson's Translation Strategies and the Resulted Quality of Indonesian Translation of Hyperboles in The Shrunken Head Novel. 
Gunawan, F. (2013). Translation Errors in English-Indonesian Humor Text Produced by Student of Basic Translation Class.

Aliaga and Gunderson. (2002). Quantitative Research Approach. Thousand Oaks, CA: Sage Publication. 